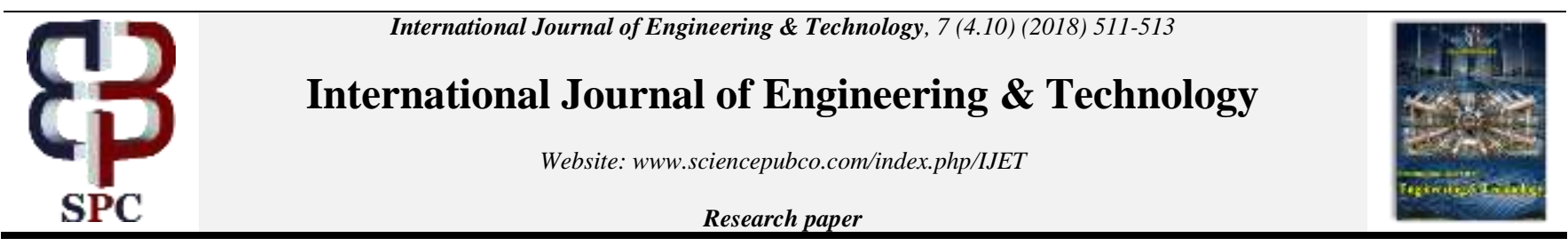

\title{
A Study on Regular Semigroups and its Idempotents
}

\author{
Julie Thomas $^{1}$, K. Indhira ${ }^{2}$, V.M. Chandrasekaran ${ }^{3 *}$ \\ Department of Mathematics, School of Advanced Sciences, VIT, Vellore, India \\ *Corresponding author E-mail: vmcsn@vit.ac.in
}

\begin{abstract}
An idempotent of a semigroup $T$ is an element $e$ in $T$ such that $e^{2}=e$. In many semigroups, idempotents can be recognized easily. Thus it plays an important role in the structure of semigroups especially on regular semigroups. This article reviews about some research work done about the structure of regular semigroups with a special emphasis on its idempotents.
\end{abstract}

Keywords: Medial idempotent; Middle unit; Normal idempotent; Regular idempotent; Quasi-medial idempotent.

\section{Introduction}

Regular semigroups can be considered as the core semigroups since groups are regular semigroups with a unique idempotent. The idempotents play a predominant role in the structure of regular semigroups. Several authors have extensively studied about its properties. The set $E$ of idempotents of a semigroup $T$ can be partially ordered by defining $e \leq f$ if and only if $e f=f e=e$. In 1972, Fitz Gerald deduced that the subsemigroup generated by the idempotents of a regular semigroup is itself regular.

Different classes of regular semigroups were defined based on some properties of idempotents. Inverse semigroups, orthodox semigroups, locally inverse semigroups, idempotent generated regular semigroups, etc are some of them. Inverse semigroups were introduced independently by Wagner (1952) and by Preston (1954). A semigroup $T$ is an inverse semigroup if and only if it is regular and its idempotents commute. If $T$ is an inverse semigroup then the partial order on $E$ reduces to $e f=e$ and $E$ then becomes a semilattice, with $e \wedge f=e f$.

Locally inverse semigroups and orthodox semigroups are regular generalisations of inverse semigroups. A regular semigroup $T$ with set $E$ of idempotents is called locally inverse if $e T e$ is an inverse semigroup for every $e$ in $E$. An orthodox semigroup is a regular semigroup in which the idempotents form a subsemigroup. This class of semigroups is due to Hall (1969) and Yamada (1970). In an orthodox semigroup $T$, the elements $a e a^{\prime}, a^{\prime} e a$ and $e^{\prime}$ are idempotents for all $a \in T, e \in E, a^{\prime} \in V(a)$ and $e^{\prime} \in V(e)$.

A band (a semigroup in which every element is an idempotent) $B$ is (left, resp. right) regular if it satisfies the identity (efe $=e f$, resp. $e f e=f e$ ) efege $=$ efge. Similarly, a band $B$ is called (left, resp. right) normal if it satisfies the identity $(a e f=a f e$, resp. efa=fea) $a e f b=$ afeb. If each $R$-class of a regular semigroup contains a unique idempotent, then it is said to be $R$-unipotent. The class of $L$-unipotent semigroups can be defined dually. Venkatesan (1974) proved that $R$-unipotent semigroups are orthodox with a left regular band of idempotents. $R$-unipotent semigroups can be redefined as a regular semigroup $T$ in which the set of idempotents forms a left regular band.
Several researchers have studied the structure of regular semigroups based on certain identities satisfied by the set of idempotents. Chrislock (1969) dealt with semigroups which satisfy the identity $x a b y=x b a y$, called the medial semigroups and showed that regular medial semigroups are those semigroups which are orthodox normal bands of abelian groups. Yamada (1967) found the structure of generalized inverse semigroup. A generalized inverse semigroup is a regular semigroup in which the set of idempotents satisfies a permutation identity $x_{1} x_{2} \ldots x_{n}=x_{p 1} x_{p 2} \ldots x_{p n}$ where $\left(p_{1}, p_{2}, \ldots, p_{n}\right)$ is a non-trivial permutation of $(1,2, \ldots, n)$.

\section{Theorem 1}

The following conditions on a regular semigroup $\mathrm{T}$ are equivalent:

(i) $\mathrm{T}$ is a generalized inverse semigroup.

(ii) The set of idempotents of $\mathrm{T}$ is a normal band.

(iii) $\mathrm{T}$ is orthodox and locally inverse semigroup.

Thus, a generalised inverse semigroup can be redefined as a regular semigroup $T$ with its idempotents form a normal band. Yamada (1973) studied about the structure of orthodox semigroups whose idempotents satisfy the identity $a b a c a=a b c a$. The set of idempotents of an orthodox semigroup $T$ satisfies $a b a c a=a b c a$ if and only if $T$ is isomorphic to a subdirect product of a left inverse semigroup $(a b a=a b, \forall a, b \in E)$ and a right inverse semigroup $(a b a=b a, \forall a, b \in E)$.

By giving some conditions on idempotents of a regular semigroup to obtain idempotents such as medial, weak medial, normal, quasinormal, middle unit, weak middle unit, one-sided identity, identity etc we will get some other classes of regular semigroups. A semigroup has a middle unit $u$ if $a u b=a b$ for all $a$ and $b$.

An idempotent $u$ of a regular semigroup $T$ will be called a weak middle unit if $(\forall x \in T)\left(\exists x^{\prime} \in V(x)\right) x u x^{\prime}=x x^{\prime}$ and $x^{\prime} u x=x^{\prime} x$. Blyth and Almeida Santos (1993) have shown that the existential quantifier can be replaced by a universal quantifier when $u$ is the biggest idempotent of $T$. Janet E. Ault (1974) proved the following theorem.

\section{Theorem 2}

Let $\mathrm{T}$ be a regular semigroup with middle units. Then 
(i) The middle units of $\mathrm{T}$ form a band, and the middle units are maximal idempotents of $\mathrm{T}$.

(ii) If e is a middle unit, then $f$ is a middle unit if and only if $f$ is an inverse of $\mathrm{e}$.

(iii) If $\mathrm{x}$ has an inverse $\mathrm{x}^{\prime}$ with $\mathrm{xx}^{\prime}\left(\mathrm{x}^{\prime} \mathrm{x}\right)$ a middle unit, then $\mathrm{xy}(\mathrm{yx})$ is a middle unit for any inverse $\mathrm{y}$ of $\mathrm{x}$.

McAlister (1981) has shown that $T$ is both naturally ordered and orthodox if and only if the biggest idempotent $u$ is a middle unit McAlister and McFadden (1983) have shown that a regular semigroup can be naturally ordered in such a way that it has a biggest idempotent if and only if it is locally inverse and contains a weak middle unit. Blyth and McFadden (1983) introduced a new type of idempotent in a regular semigroup called medial idempotent. An idempotent $u$ of a regular semigroup $T$ will be called medial if it is such that $(\forall x \in \bar{E}) x=x u x$ where $\bar{E}$ is the subsemigroup generated by the set $E$ of idempotents of $T$. Thus every medial idempotent is a weak middle unit.

A middle unit of a band is medial. Also if $u$ is a medial idempotent then $\bar{E} u, u \bar{E}$ and $u \bar{E} u$ are subbands of $T$. As a result, the subsemigroup $\bar{E}$ of $T$ containing a medial idempotent is periodic and combinatorial. A medial idempotent $u$ will be called normal if the subband $u \bar{E} u$ is commutative. Blyth and McFadden have got a condition under which a regular semigroup which contains a normal idempotent is orthodox.

\section{Theorem 3}

Let $\mathrm{T}$ be a regular semigroup containing a normal idempotent $\mathrm{u}$. Then

(i) $\mathrm{T}$ is orthodox if and only if $\mathrm{u}$ is a middle unit.

(ii) $\mathrm{T}$ is inverse if and only if $\mathrm{u}$ is an identity element.

Further, Blyth and McFadden have described idempotent- generated regular semigroups that contain a medial idempotent in terms of bands with one sided identities. Also, they have described a method for constructing all regular semigroups that contain a normal idempotent in terms of idempotent-generated regular semigroups with an identity.

Loganathan (1987) has constructed a semigroup $W=W(\bar{E}, T)$ in terms of idempotent-generated regular semigroup $\bar{E}$, with a medial idempotent $u$, and of the orthodox semigroups with identity $T$, such that $E \cong u \bar{E} u$. Thus he has modified method of [4] and showed that every regular semigroup $T$ with a medial idempotent $u$ is of the form $W$. Later Hysa (2012) studied about $W=W(\bar{E}, T)$ constructed by Loganathan and proved that it is a regular semigroup with a medial idempotent. S. Hussain, T. Anwer and H. Chien (2010) have studied the properties of regular semigroups that contains a medial idempotent. Xiangfei Ni and Haizhou Chao (2017) have tried to characterize normal idempotent in different ways. They have obtained a non-orthodox non-idempotent generated regular semigroup which contains a normal idempotent and investigated different properties of a regular semigroup having a normal idempotent.

\section{Theorem 4}

An idempotent $\mathrm{u}$ of a regular semigroup $\mathrm{T}$ is normal if and only if uTu is a multiplicative inverse transversal for T. Also, the identity of a multiplicative inverse transversal $T^{0}$ of $\mathrm{T}$ is a normal idempotent of $\mathrm{T}$.

Since a regular semigroup $T$ with an inverse transversal is locally inverse if and only if it is a quasi-ideal of $T$ [5], we will get that a regular semigroup which contains a normal idempotent is locally inverse.

\section{Theorem 5}

Let $\mathrm{u}$ be a normal idempotent of a regular semigroup $\mathrm{T}$. Then the following are equivalent.

(i) $\mathrm{T}$ is an orthodox semigroup. (ii) $(\forall x \in T) u x u \in E \Rightarrow x \in E$.

(iii) $(\forall e, f \in E)$ uefu $\in E \Rightarrow e f \in E$.

Thus the above conditions are equivalent to $u$ being a middle unit by Theorem 3. The building bricks used by Blyth and McFadden for constructing every regular semigroup that contains a normal idempotent, were the idempotent generated regular semigroup and an inverse semigroup with an identity. But Xiangfei $\mathrm{Ni}$ and Haizhou Chao have characterized the same by means of a left inverse semigroup and a right inverse semigroup.

Blyth and Almeida Santos (2002) introduced the notion of skew pair in the sense that an ordered pair $(e, f)$ of idempotents of a regular semigroup is called a skew pair if $e f \notin E$ whereas $f e \in E$. Indhira and Chandrasekaran (2011) introduced the concept of regular idempotent and studied properties of regular semigroups with regular idempotent. An idempotent $u$ of $T$ is called a regular idempotent if $f u \mathcal{R} f \mathcal{L} u f$ for each $f \in E$. If $u$ is a regular idempotent of $T$ then $T$ can be described in terms of $T^{0}=u T u$ which is a regular subsemigroup of $T$. Now, if $u$ is a medial idempotent, then $f u f=f \cdot f=f$ implies $f u \mathcal{R} f \mathcal{L} u f$ for each $f \in E$ and therefore $u$ becomes a regular idempotent of $T$. Thus the class of regular semigroups having a regular idempotent includes the class of regular semigroups with a medial idempotent as well as with a middle unit.

Xiangfei Ni, Haizhou Chao (2016) have introduced the concept of a quasi-normal idempotent of $T$. An idempotent element $u$ of $T$ is said to be quasi-normal idempotent if $e u e=e$ for every $e \in E$ and $u E u$ is a semilattice. Thus every normal idempotent is a quasinormal idempotent. They have shown that the converse is also true. Xiangfei Ni and Xiao Jiang Guo (2018) have introduced the concepts of weak medial idempotent and quasi-medial idempotent of regular semigroups and explored some of the properties of these idempotents. They have constructed several regular semigroups having weak medial idempotents and quasi-medial idempotents and obtained the structure theorem for all regular semigroups with a quasi-medial idempotent which will help to determine whether a regular semigroup contains a multiplicative inverse transversal or not.

Thus the idempotents are seen to be immensely useful in the study of the structure of regular semigroups. We have obtained several classes of regular semigroups by giving different types of restrictions on the set of idempotents and this lead us to complete determination of the structure. The concepts discussed above can be extended in different ways not only in regular semigroups but also to some other classes of semigroups and the researches are still going on.

\section{References}

[1] G.R. Baird, "Congruences on generalized inverse semigroups", Semigroup Forum, Vol.4, (1972), pp.200-205, available online: https://doi.org/10.1007/BF02570786

[2] T.S. Blyth, "On middle units in orthodox semigroups", Semigroup Forum, Vol.13, (1977), pp.261-265, available online: https://doi.org/10.1007/BF02194944

[3] T.S. Blyth \& R. McFadden, "Naturally ordered regular semigroups with a greatest idempotent", Proc. Roy. Soc. Edinburgh, Vol.91A, (1981), pp.107-122, available online: https://doi.org/10.1017/S0308210500012671

[4] T.S. Blyth \& R. McFadden, "On the construction of a class of regular semigroups", J. Algebra, Vol.81, (1983), pp.1-22, available online: https://doi.org/10.1016/0021-8693(83)90205-3

[5] T.S. Blyth \& M.H. Almeida Santos, "On naturally ordered regular semigroups with biggest idempotents", Comm. Algebra, Vol.21, No.5, (1993), pp.1761-1771, available online: https://doi.org/10.1080/00927879308824651

[6] T.S. Blyth \& M.H. Almeida Santos, "A classification of inverse transversals”, Comm. Algebra, Vol.29, No.2, (2001), pp.611-624, available online: https://doi.org/10.1081/AGB-100001527

[7] T.S. Blyth \& M.H. Almeida Santos, "Regular semigroups with skew pairs of idempotents", Semigroup Forum, Vol.65, (2002), 
pp.264-274,

available https://doi.org/10.1007/s002330010112

[8] T.S. Blyth \& M.H. Almeida Santos, "Naturally ordered regular semigroups with inverse monoid transversal", Semigroup Forum, Vol.76, (2008), pp.71-86, available online: https://doi.org/10.1007/s00233-007-9002-z

[9] J.L. Chrislock, "On medial semigroups", J. Algebra, Vol.12, (1969), pp.1-9, available online: https://doi.org/10.1016/00218693(69)90013-1

[10] A.H. Clifford \& G.B. Preston, The Algebraic Theory of Semigroups Vol. 1, Math. Surveys of the American Math. Soc. 7, Providence, Rhode Island, 1961

[11] D.G. Fitz-Gerald, "On inverses of products of idempotents in regular semigroups", J. Austral. Math. Soc., Vol.13, (1972), pp.335-337, available online: https://doi.org/10.1017/S1446788700013756

[12] X.J. Guo, "The structure of abundant semigroups with a weak normal idempotent", Acta Mathematics Sinica, Vol.42, No.4, (1999), pp.683-690.

[13] T.E. Hall, "On regular semigroups whose idempotents form a subsemigroup”, Bull. Austral. Math. Soc., Vol.1, (1969), pp.195-208, available online: https://doi.org/10.1017/S0004972700041447

[14] T.E. Hall, “On regular semigroups”, J. Algebra, Vol.24, (1973) pp.1-24, available online: https://doi.org/10.1016/00218693(73)90150-6

[15] J.M. Howie, "Fundamentals of semigroup theory", Oxford University Press, New York, 1995.

[16] S. Hussain, T. Anwer \& H. Chien, "Some properties of regula semigroups possess a medial idempotent", Int. J. Algebra, Vol.4, No.9, (2010), pp.433-438.

[17] O. Hysa, "The construction of regular semigroups with medial idempotent", International Journal of Basic and Applied Sciences IJBAS-IJENS, Vol.12, No.6, (2012), pp.49-52.

[18] K. Indhira \& V.M. Chandrasekaran, "Structure of regular semigroups with a regular idempotent", Int. J. Contemp. Math. Sciences, Vol.6, No. 12, (2011), pp.557-570.

[19] K. Indhira \& V.M. Chandrasekaran, "Construction of generalized inverse semigroups", Int. J. lgebra, Vol.5, No.15, (2011), pp.739746.

[20] K. Indhira \& V.M. Chandrasekaran, "Idempotent separating congruence on a regular semigroup with a regular idempotent", Adv Studies Theor. Phys., Vol.7, No.3, (2013), pp.107-114.

[21] Janet E. Ault, "Semigroups with midunits", Trans. Amer. Math Soc., Vol.190, (1974), pp.375-384, available online: https://doi.org/10.1007/BF02389143

[22] G. Kudryavtseva \& M.V. Lawson, "The structure of generalized inverse semigroups", Semigroup Forum, Vol.89, No.1, (2014), pp.199-216, available online: https://doi.org/10.1007/s00233-013 9518-3

[23] M. Loganathan, "Regular semigroup with a medial idempotent", Semigroup Forum, Vol.36, (1987), pp.69-74, available online: https://doi.org/10.1007/BF02575006

[24] S. Madhavan, "Some results on generalized inverse semigroups", Semigroup Forum, Vol.16, (1978), pp.355-367, available online: https://doi.org/10.1007/BF02194635

[25] Mario Petrich, "Regular semigroups satisfying certain conditions on idempotents and ideals", Trans. Amer. Math. Soc., Vol.170, (1972) pp. $245-267$

[26] D.B. McAlister, "Regular Rees matrix semigroups and regular Dubreil-Jacotin semigroups", J. Australian Math. Soc., Vol.31, (1981), pp.325-336, available online: https://doi.org/10.1017/S1446788700019467

[27] D.B. McAlister \& R. McFadden, "Maximum idempotents in naturally ordered regular semigroups", Proc. Edinburgh Math. Soc., Vol.26, (1983), pp.213-220, available online: https://doi.org/10.1017/S0013091500016916

[28] H.E. Scheiblich, "Generalized inverse semigroups with involution", Rocky Mountain J. Math., Vol.12, No.2, (1982), pp.205-211.

[29] P.S. Venkatesan, "Right (left) inverse semigroups", J. Algebra, Vol.31, (1974), pp.209-217, available online: https://doi.org/10.1016/0021-8693(74)90064-7

[30] Xiangfei Ni \& Haizhou Chao, "A note on normal idempotents", Publicationes Mathematicae, Vol.89, No.4, (2016), pp.441-448.

[31] Xiangfei Ni \& Haizhou Chao, "Regular semigroups with normal idempotents", J. Australian Math. Soc., Vol.103, No.1, (2017), pp.116-125, available online: https://doi.org/10.1017/S1446788717000088
[32] Xiangfei Ni \& Xiao Jiang Guo, "Regular semigroups with weak medial idempotents", Acta Mathematica Sinica, Vol.61, No.1, (2018), pp.107-122.

[33] M. Yamada, "Regular semigroups whose idempotents satisfy permutation identities", Pacific. J. Math., Vol.21, (1967), pp.371-392.

[34] M. Yamada, "On a regular semigroup in which the idempotents form a band”, Pacific J. Math., Vol.33, (1970), pp.251-272.

[35] M. Yamada, "Orthodox semigroups whose idempotents satisfy a certain identity”, Semigroup Forum, Vol.6, No.1, (1973), 113-128, available online: https://doi.org/10.1007/BF02389116 